\title{
Abstract fractional integro-differential equations involving nonlocal initial conditions in $\alpha$-norm
}

\author{
Rong-Nian Wang ${ }^{*}$, Jun Liu and De-Han Chen
}

* Correspondence: rnwang@mail. ustc.edu.cn

Department of Mathematics, NanChang University, NanChang, JiangXi 330031, People's Republic of China

\begin{abstract}
In the present paper, we deal with the Cauchy problems of abstract fractional integro-differential equations involving nonlocal initial conditions in $\alpha$-norm, where the operator $A$ in the linear part is the generator of a compact analytic semigroup. New criterions, ensuring the existence of mild solutions, are established. The results are obtained by using the theory of operator families associated with the function of Wright type and the semigroup generated by $A$, Krasnoselkii's fixed point theorem and Schauder's fixed point theorem. An application to a fractional partial integrodifferential equation with nonlocal initial condition is also considered.

Mathematics subject classification (2000)

26A33, 34G10, 34G20
\end{abstract}

Keywords: Cauchy problem of abstract fractional evolution equation, Nonlocal initial condition, Fixed point theorem, Mild solution, $a$-norm

\section{Introduction}

Let $(A, D(A))$ be the infinitesimal generator of a compact analytic semigroup of bounded linear operators $\{T(t)\}_{t \geq 0}$ on a real Banach space $(X,\|\cdot\|)$ and $0 \in \rho(A)$. Denote by $X_{\alpha}$, the Banach space $D\left(A^{\alpha}\right)$ endowed with the graph norm $\|u\|_{\alpha}=\left\|A^{\alpha} u\right\|$ for $u \in X_{\alpha}$. The present paper concerns the study of the Cauchy problem for abstract fractional integro-differential equation involving nonlocal initial condition

$$
\left\{\begin{aligned}
{ }^{c} D_{t}^{\beta} u(t)=A u(t) & +F\left(t, u(t), u\left(\kappa_{1}(t)\right)\right) \\
& +\int_{0}^{t} K(t-s) G\left(s, u(s), u\left(\kappa_{2}(s)\right)\right) \mathrm{d} s, \quad t \in[0, T], \\
u(0)+H(u)=u_{0} &
\end{aligned}\right.
$$

in $X_{\alpha}$, where ${ }^{c} D_{t}^{\beta}, 0<\beta<1$, stands for the Caputo fractional derivative of order $\beta$, and $K:[0, T] \rightarrow \mathbb{R}^{+}, \kappa_{1}, \kappa_{2}:[0, T] \rightarrow[0, T], F, G:[0, T] \times X_{\alpha} \times X_{\alpha} \rightarrow X, H: C([0, T]$; $\left.X_{\alpha}\right) \rightarrow X_{\alpha}$ are given functions to be specified later. As can be seen, $H$ constitutes a nonlocal condition.

The fractional calculus that allows us to consider integration and differentiation of any order, not necessarily integer, has been the object of extensive study for analyzing not only anomalous diffusion on fractals (physical objects of fractional dimension, like some amorphous semiconductors or strongly porous materials; see [1-3] and references therein), but also fractional phenomena in optimal control (see, e.g., [4-6]). As indicated in $[2,5,7]$ and the related references given there, the advantages of fractional

(c) 2011 Wang et al; licensee Springer. This is an Open Access article distributed under the terms of the Creative Commons Attribution License (http://creativecommons.org/licenses/by/2.0), which permits unrestricted use, distribution, and reproduction in any medium, provided the original work is properly cited. 
derivatives become apparent in modeling mechanical and electrical properties of real materials, as well as in the description of rheological properties of rocks, and in many other fields. One of the emerging branches of the study is the Cauchy problems of abstract differential equations involving fractional derivatives in time. In recent decades, there has been a lot of interest in this type of problems, its applications and various generalizations (cf. e.g., [8-11] and references therein). It is significant to study this class of problems, because, in this way, one is more realistic to describe the memory and hereditary properties of various materials and processes (cf. $[4,5,12,13])$.

In particular, much interest has developed regarding the abstract fractional Cauchy problems involving nonlocal initial conditions. For example, by using the fractional power of operators and some fixed point theorems, the authors studied the existence of mild solutions in [14] for fractional differential equations with nonlocal initial conditions and in [15] for fractional neutral differential equations with nonlocal initial conditions and time delays. The existence of mild solutions for fractional differential equations with nonlocal initial conditions in $\alpha$-norm using the contraction mapping principle and the Schauder's fixed point theorem have been investigated in [16].

We here mention that the abstract problem with nonlocal initial condition was first considered by Byszewski [17], and the importance of nonlocal initial conditions in different fields has been discussed in [18,19] and the references therein. Deng [19], especially, gave the following nonlocal initial values: $H(u)=\sum_{i=1}^{p} C_{i} u\left(t_{i}\right)$, where $C_{i}(i=1$, $\ldots, p)$ are given constants and $0<t_{1}<\cdots<t_{p-1}<t_{p}<+\infty(p \in \mathbb{N})$, which is used to describe the diffusion phenomenon of a small amount of gas in a transparent tube. In the past several years theorems about existence, uniqueness and stability of Cauchy problem for abstract evolution equations with nonlocal initial conditions have been studied by many authors, see for instance [19-28] and references therein.

In this paper, we will study the existence of mild solutions for the fractional Cauchy problem (1.1). New criterions are established. Both Krasnoselkii's fixed point theorem and Schauder's fixed point theorem, and the theory of operator families associated with the function of Wright type and the semigroup generated by $A$, are employed in our approach. The results obtained are generalizations and continuation of the recent results on this issue.

The paper is organized as follows. In Section 2, some required notations, definitions and lemmas are given. In Section 3, we present our main results and their proofs.

\section{Preliminaries}

In this section, we introduce some notations, definitions and preliminary facts which are used throughout this work.

We first recall some definitions of fractional calculus (see e.g., [6,13] for more details).

Definition 2.1 The Riemann-Liouville fractional integral operator of order $\beta>0$ of function $f$ is defined as

$$
I^{\beta} f(t)=\frac{1}{\Gamma(\beta)} \int_{0}^{t}(t-s)^{\beta-1} f(s) \mathrm{d} s,
$$


provided the right-hand side is pointwise defined on $[0, \infty)$, where $\Gamma(\cdot)$ is the gamma function.

Definition 2.2 The Caputo fractional derivative of order $\beta>0, m-1<\beta<m, m \in \mathbb{N}$, is defined as

$$
{ }^{c} D^{\beta} f(t)=I^{m-\beta} D_{t}^{m} f(t)=\frac{1}{\Gamma(m-\beta)} \int_{0}^{t}(t-s)^{m-\beta-1} D_{s}^{m} f(s) \mathrm{d} s,
$$

where $D_{t}^{m}:=\frac{d^{m}}{\mathrm{~d} t^{m}}$ and $f$ is an abstract function with value in $X$. If $0<\beta<1$, then

$$
{ }^{c} D^{\beta} f(t)=\frac{1}{\Gamma(1-\beta)} \int_{0}^{t} \frac{f^{\prime}(s)}{(t-s)^{\beta}} \mathrm{d} s .
$$

Throughout this paper, we let $A: D(A) \rightarrow X$ be the infinitesimal generator of a compact analytic semigroup of bounded linear operators $\{T(t)\}_{t \geq 0}$ on $X$ and $0 \in \rho(A)$, which allows us to define the fractional power $A^{\alpha}$ for $0 \leq \alpha<1$, as a closed linear operator on its domain $D\left(A^{\alpha}\right)$ with inverse $A^{-\alpha}$.

Let $X_{\alpha}$ denote the Banach space $D\left(A^{\alpha}\right)$ endowed with the graph norm $\|u\|_{\alpha}=\|$ $A^{\alpha} u \|$ for $u \in X_{\alpha}$ and let $C\left([0, T] ; X_{\alpha}\right)$ be the Banach space of all continuous functions from $[0, T]$ into $X_{\alpha}$ with the uniform norm topology

$$
|u|_{\alpha}=\sup \left\{\|u(t)\|_{\alpha}, t \in[0, T]\right\} .
$$

$\mathcal{L}(X)$ stands for the Banach space of all linear and bounded operators on $X$. Let $M$ be a constant such that

$$
M=\sup \left\{\|T(t)\|_{\mathcal{L}(X)}, t \in[0, \infty)\right\} .
$$

For $k>0$, write

$$
\Theta_{k}=\left\{u \in C\left([0, T] ; X_{\alpha}\right) ;|u|_{\alpha} \leq k\right\} .
$$

The following are basic properties of $A^{\alpha}$.

Theorem 2.1 ([29], pp. 69-75)).

(a) $T(t): X \rightarrow X_{\alpha}$ for each $t>0$, and $A^{\alpha} T(t) x=T(t) A^{\alpha} x$ for each $x \in X_{\alpha}$ and $t \geq 0$.

(b) $A^{\alpha} T(t)$ is bounded on $X$ for every $t>0$ and there exist $M_{\alpha}>0$ and $\delta>0$ such that

$$
\left\|A^{\alpha} T(t)\right\|_{\mathcal{L}(X)} \leq \frac{M_{\alpha}}{t^{\alpha}} e^{-\delta t} .
$$

(c) $A^{-\alpha}$ is a bounded linear operator in $X$ with $D\left(A^{\alpha}\right)=\operatorname{Im}\left(A^{-\alpha}\right)$.

(d) If $0<\alpha_{1} \leq \alpha_{2}$, then $X_{\alpha_{2}} \cdot \nabla X_{\alpha_{1}}$

Lemma 2.1. [27] The restriction of $T(t)$ to $X_{\alpha}$ is exactly the part of $T(t)$ in $X_{\alpha}$ and is an immediately compact semigroup in $X_{\alpha}$, and hence it is immediately normcontinuous. 
Define two families $\left\{\mathcal{S}_{\beta}(t)\right\}_{t \geq 0}$ and $\left\{\mathcal{P}_{\beta}(t)\right\}_{t \geq 0}$ of linear operators by

$$
\mathcal{S}_{\beta}(t) x=\int_{0}^{\infty} \Psi_{\beta}(s) T\left(t^{\beta} s\right) x \mathrm{~d} s, \quad \mathcal{P}_{\beta}(t) x=\int_{0}^{\infty} \beta s \Psi_{\beta}(s) T\left(t^{\beta} s\right) x \mathrm{~d} s
$$

for $x \in X, t \geq 0$, where

$$
\Psi_{\beta}(s)=\frac{1}{\pi \beta} \sum_{n=1}^{\infty}(-s)^{n-1} \frac{\Gamma(1+\beta n)}{n !} \sin (n \pi \beta), \quad s \in(0, \infty)
$$

is the function of Wright type defined on $(0, \infty)$ which satisfies

$$
\begin{aligned}
& \Psi_{\beta}(s) \geq 0, \quad s \in(0, \infty), \quad \int_{0}^{\infty} \Psi_{\beta}(s) \mathrm{d} s=1, \text { and } \\
& \int_{0}^{\infty} s^{\zeta} \Psi_{\beta}(s) \mathrm{d} s=\frac{\Gamma(1+\zeta)}{\Gamma(1+\beta \zeta)}, \quad \zeta \in(-1, \infty) .
\end{aligned}
$$

The following lemma follows from the results in [15].

Lemma 2.2. The following properties hold:

(1) For every $t \geq 0, \mathcal{S}_{\beta}(t)$ and $\mathcal{P}_{\beta}(t)$ are linear and bounded operators on $X$, i.e.,

$$
\left\|\mathcal{S}_{\beta}(t) x\right\| \leq M\|x\|, \quad\left\|\mathcal{P}_{\beta}(t) x\right\| \leq \frac{\beta M}{\Gamma(1+\beta)}\|x\|
$$

for all $x \in X$ and $0 \leq t<\infty$.

(2) For every $x \in X, t \rightarrow \mathcal{S}_{\beta}(t) x, t \rightarrow \mathcal{P}_{\beta}(t) x a r e$ continuous functions from $[0, \infty)$ into $X$.

(3) $\mathcal{S}_{\beta}(t)$ and $\mathcal{P}_{\beta}(t)$ are compact operators on $X$ for $t>0$.

(4) For all $x \in X$ and $t \in(0, \infty),\left\|A^{\alpha} \mathcal{P}_{\beta}(t) x\right\| \leq C_{\alpha} t^{-\alpha \beta}\|x\|$ where $C_{\alpha}=\frac{M_{\alpha} \beta \Gamma(2-\alpha)}{\Gamma(1+\beta(1-\alpha))}$.

We can also prove the following criterion.

Lemma 2.3. The functions $t \rightarrow A^{\alpha} \mathcal{P}_{\beta}(t)$ and $t \rightarrow A^{\alpha} \mathcal{S}_{\beta}(t)$ are continuous in the uniform operator topology on $(0,+\infty)$.

Proof. Let $\varepsilon>0$ be given. For every $r>0$, from (2.1), we may choose $\delta_{1}, \delta_{2}>0$ such that

$$
\frac{M_{\alpha}}{r^{\alpha \beta}} \int_{0}^{\delta_{1}} \Psi_{\beta}(s) s^{-\alpha} \mathrm{d} s \leq \frac{\varepsilon}{6}, \quad \frac{M_{\alpha}}{r^{\alpha \beta}} \int_{\delta_{2}}^{\infty} \Psi_{\beta}(s) s^{-\alpha} \mathrm{d} s \leq \frac{\varepsilon}{6} .
$$

Then, we deduce, in view of the fact $t \rightarrow A^{\alpha} T(t)$ that is continuous in the uniform operator topology on $(0, \infty)$ (see [[30], Lemma 2.1]), that there exists a constant $\delta>$ such that

$$
\int_{\delta_{1}}^{\delta_{2}} \Psi_{\beta}(s)\left\|A^{\alpha} T\left(t_{1}^{\beta} s\right)-A^{\alpha} T\left(t_{2}^{\beta} s\right)\right\|_{\mathcal{L}(X)} \mathrm{d} s \leq \frac{\varepsilon}{3},
$$

for $t_{1}, t_{2} \geq r$ and $\left|t_{1}-t_{2}\right|<\delta$. 
On the other hand, for any $x \in X$, we write

$$
\begin{aligned}
\mathcal{S}_{\beta}\left(t_{1}\right) x-\mathcal{S}_{\beta}\left(t_{2}\right) x=\quad & \int_{0}^{\delta_{1}} \Psi_{\beta}(s)\left(T\left(t_{1}^{\beta} s\right) x-T\left(t_{2}^{\beta} s\right) x\right) \mathrm{d} s \\
& +\int_{\delta_{1}}^{\delta_{2}} \Psi_{\beta}(s)\left(T\left(t_{1}^{\beta} s\right) x-T\left(t_{2}^{\beta} s\right) x\right) \mathrm{d} s \\
& +\int_{\delta_{2}}^{\infty} \Psi_{\beta}(s)\left(T\left(t_{1}^{\beta} s\right) x-T\left(t_{2}^{\beta} s\right) x\right) \mathrm{d} s .
\end{aligned}
$$

Therefore, using $(2.2,2.3)$ and Lemma 2.2, we get

$$
\begin{aligned}
& \left\|A^{\alpha} \mathcal{S}_{\beta}\left(t_{1}\right) x-A^{\alpha} \mathcal{S}_{\beta}\left(t_{2}\right) x\right\| \\
& \leq \int_{0}^{\delta_{1}} \Psi_{\beta}(s)\left(\left\|A^{\alpha} T\left(t_{1}^{\beta} s\right)\right\|_{\mathcal{L}(X)}+\left\|A^{\alpha} T\left(t_{2}^{\beta} s\right)\right\|_{\mathcal{L}(X)}\right)\|x\| \mathrm{d} s \\
& \quad+\int_{\delta_{1}}^{\delta_{2}} \Psi_{\beta}(s)\left\|A^{\alpha} T\left(t_{1}^{\beta} s\right)-A^{\alpha} T\left(t_{2}^{\beta} s\right)\right\|_{\mathcal{L}(X)}\|x\| \mathrm{d} s \\
& \quad+\int_{\delta_{2}}^{\infty} \Psi_{\beta}(s)\left(\left\|A^{\alpha} T\left(t_{1}^{\beta} s\right)\right\|_{\mathcal{L}(X)}+\left\|A^{\alpha} T\left(t_{2}^{\beta} s\right)\right\|_{\mathcal{L}(X)}\right)\|x\| \mathrm{d} s \\
& \leq \frac{2 M_{\alpha}}{r^{\alpha \beta}} \int_{0}^{\delta_{1}} \Psi_{\beta}(s) s^{-\alpha}\|x\| \mathrm{d} s \\
& +\int_{\delta_{1}}^{\delta_{2}} \Psi_{\beta}(s)\left\|T\left(t_{1}^{\beta} s\right)-T\left(t_{2}^{\beta} s\right)\right\|_{\mathcal{L}(X)}\|x\| \mathrm{d} s \\
& +\frac{2 M_{\alpha}}{r^{\alpha \beta}} \int_{\delta_{2}}^{\infty} \Psi_{\beta}(s) s^{-\alpha}\|x\| \mathrm{d} s \\
& \leq \varepsilon\|x\|,
\end{aligned}
$$

that is,

$$
\left\|A^{\alpha} \mathcal{S}_{\beta}\left(t_{1}\right)-A^{\alpha} \mathcal{S}_{\beta}\left(t_{2}\right)\right\| \leq \varepsilon, \quad \text { for } t_{1}, t_{2} \geq r \text { and }\left|t_{1}-t_{2}\right|<\delta
$$

which together with the arbitrariness of $r>0$ implies that $A^{\alpha} \mathcal{P}_{\beta}(t)$ is continuous in the uniform operator topology for $t>0$. A similar argument enable us to give the characterization of continuity on $A^{\alpha} \mathcal{P}_{\beta}(t)$. This completes the proof.

Lemma 2.4. For every $t>0$, the restriction of $\mathcal{S}_{\beta}(t)$ to $X_{\alpha}$ and the restriction of $\mathcal{P}_{\beta}(t)$ to $X_{\alpha}$ are compact operators in $X_{\alpha}$.

Proof. First consider the restriction of $\mathcal{S}_{\beta}(t)$ to $X_{\alpha}$. For any $r>0$ and $t>0$, it is sufficient to show that the set $\left\{\mathcal{S}_{\beta}(t) u ; u \in B_{r}\right\}$ is relatively compact in $X_{\alpha}$, where $B_{r}:=\{u \in$ $\left.X_{\alpha} ;\|u\|_{\alpha} \leq r\right\}$.

Since by Lemma 2.1, the restriction of $T(t)$ to $X_{\alpha}$ is compact for $t>0$ in $X_{\alpha}$, for each $t>0$ and $\varepsilon \in(0, t)$,

$$
\begin{aligned}
& \left\{\int_{\varepsilon}^{\infty} \Psi_{\beta}(s) T\left(t^{\beta} s\right) u \mathrm{~d} s ; u \in B_{r}\right\} \\
& =\left\{T\left(t^{\beta} \varepsilon\right) \int_{\varepsilon}^{\infty} \Psi_{\beta}(s) T\left(t^{\beta} s-t^{\beta} \varepsilon\right) u \mathrm{~d} s ; u \in B_{r}\right\}
\end{aligned}
$$


is relatively compact in $X_{\alpha}$. Also, for every $u \in B_{r}$, as

$$
\int_{\varepsilon}^{\infty} \Psi_{\beta}(s) T\left(t^{\beta} s\right) u \mathrm{~d} s \rightarrow \mathcal{S}_{\beta}(t) u, \quad \varepsilon \rightarrow 0
$$

in $X_{\alpha}$, we conclude, using the total boundedness, that the set $\left\{\mathcal{S}_{\beta}(t) u ; u \in B_{r}\right\}$ is relatively compact, which implies that the restriction of $\mathcal{S}_{\beta}(t)$ to $X_{\alpha}$ is compact. The same idea can be used to prove that the restriction of $\mathcal{P}_{\beta}(t)$ to $X_{\alpha}$ is also compact.

The following fixed point theorems play a key role in the proofs of our main results, which can be found in many books.

Lemma 2.5 (Krasnoselskii's Fixed Point Theorem). Let $E$ be a Banach space and B be a bounded closed and convex subset of $E$, and let $F_{1}, F_{2}$ be maps of $B$ into $E$ such that $F_{1} x+F_{2} y \in B$ for every pair $x, y \in B$. If $F_{1}$ is a contraction and $F_{2}$ is completely continuous, then the equation $F_{1} x+F_{2} x=x$ has a solution on $B$.

Lemma 2.6 (Schauder Fixed Point Theorem). If $B$ is a closed bounded and convex subset of a Banach space $E$ and $F: B \rightarrow B$ is completely continuous, then $F$ has a fixed point in $B$.

\section{Main results}

Based on the work in [[15], Lemma 3.1 and Definition 3.1], in this paper, we adopt the following definition of mild solution of Cauchy problem (1.1).

Definition 3.1. By a mild solution of Cauchy problem (1.1), we mean a function $u \in$ $C\left([0, T] ; X_{\alpha}\right)$ satisfying

$$
\begin{array}{r}
u(t)=\mathcal{S}_{\beta}(t)\left(u_{0}-H(u)\right)+\int_{0}^{t}(t-s)^{\beta-1} \mathcal{P}_{\beta}(t-s)\left(F\left(s, u(s), u\left(\kappa_{1}(s)\right)\right)\right. \\
\left.+\int_{0}^{s} K(s-\tau) G\left(\tau, u(\tau), u\left(\kappa_{2}(\tau)\right)\right) \mathrm{d} \tau\right) \mathrm{d} s
\end{array}
$$

for $t \in[0, T]$.

Let us first introduce our basic assumptions.

$\left(H_{0}\right) \kappa_{1}, \kappa_{2} \in C([0, T] ;[0, T])$ and $K \in C\left([0, T] ; \mathbb{R}^{+}\right)$.

$\left(H_{1}\right) F, G:[0, T] \times X_{\alpha} \times X_{\alpha} \rightarrow X$ are continuous and for each positive number $k \in$ $\mathbb{N}$, there exist a constant $\gamma \in[0, \beta(1-\alpha))$ and functions $\phi_{k}(\cdot) \in L^{1 / \gamma}\left(0, T ; \mathbb{R}^{+}\right), \varphi_{k}(\cdot)$ $\in L^{\infty}\left(0, T ; \mathbb{R}^{+}\right)$such that

$$
\begin{array}{lll}
\sup _{\|u\|_{\alpha},\|v\|_{\alpha} \leq k}\|F(t, u, v)\| \leq \varphi_{k}(t) \text { and } & \liminf _{k \rightarrow+\infty} \frac{\left\|\varphi_{k}\right\|_{L^{1 / \gamma}(0, T)}}{k}=\sigma_{1}<\infty, \\
\sup _{\|u\|_{\alpha},\|v\|_{\alpha} \leq k}\|G(t, u, v)\| \leq \phi_{k}(t) \text { and } & \liminf _{k \rightarrow+\infty} \frac{\left\|\phi_{k}\right\|_{L^{\infty}(0, T)}}{k}=\sigma_{2}<\infty .
\end{array}
$$

$\left(H_{2}\right) F, G:[0, T] \times X_{\alpha} \times X_{\alpha} \rightarrow X$ are continuous and there exist constants $L_{F}, L_{K}$ such that

$$
\begin{aligned}
& \left\|F\left(t, u_{1}, v_{1}\right)-F\left(t, u_{2}, v_{2}\right)\right\| \leq L_{F}\left(\left\|u_{1}-u_{2}\right\|_{\alpha}+\left\|v_{1}-v_{2}\right\|_{\alpha}\right), \\
& \left\|G\left(t, u_{1}, v_{1}\right)-G\left(t, u_{2}, v_{2}\right)\right\| \leq L_{G}\left(\left\|u_{1}-u_{2}\right\|_{\alpha}+\left\|v_{1}-v_{2}\right\|_{\alpha}\right)
\end{aligned}
$$


for all $\left(t, u_{1}, v_{1}\right),\left(t, u_{2}, v_{2}\right) \in[0, T] \times X_{\alpha} \times X_{\alpha}$.

$\left(H_{3}\right) H: C\left([0, T] ; X_{\alpha}\right) \rightarrow X_{\alpha}$ is Lipschitz continuous with Lipschitz constant $L_{H}$.

$\left(H_{4}\right) H: C\left([0, T] ; X_{\alpha}\right) \rightarrow X_{\alpha}$ is continuous and there is a $\eta \in(0, T)$ such that for any $u, w \in C\left([0, T] ; X_{\alpha}\right)$ satisfying $u(t)=w(t)(t \in[\eta, T]), H(u)=H(w)$.

$\left(H_{5}\right)$ There exists a nondecreasing continuous function $\Phi: \mathbb{R}^{+} \rightarrow \mathbb{R}^{+}$such that for all $u \in \Theta_{k}$,

$$
\|H(u)\|_{\alpha} \leq \Phi(k), \quad \text { and } \quad \liminf _{k \rightarrow+\infty} \frac{\Phi(k)}{k}=\mu<\infty .
$$

Remark 3.1. Let us note that $\left(H_{4}\right)$ is the case when the values of the solution $u(t)$ for $t$ near zero do not affect $H(u)$. We refer to [19]for a case in point.

In the sequel, we set $\tilde{k}:=\int_{0}^{T} K(t) \mathrm{d} t$. We are now ready to state our main results in this section.

Theorem 3.1. Let the assumptions $\left(H_{0}\right),\left(H_{1}\right)$ and $\left(H_{3}\right)$ be satisfied. Then, for $u_{0} \in$ $X_{\infty}$ the fractional Cauchy problem (1.1) has at least one mild solution provided that

$$
M L_{H}+C_{\alpha} \sigma_{1} T^{(1-\alpha) \beta-\gamma}\left(\frac{1-\gamma}{(1-\alpha) \beta-\gamma}\right)^{1-\gamma}+\frac{C_{\alpha} \sigma_{2} \tilde{k} T^{(1-\alpha) \beta}}{(1-\alpha) \beta}<1 .
$$

Proof. Let $v \in C\left([0, T] ; X_{\alpha}\right)$ be fixed with $|v|_{\alpha} \equiv 0$. From (3.1) and $\left(H_{1}\right)$, it is easy to see that there exists a $k_{0}>0$ such that

$$
\begin{gathered}
M\left(\left\|u_{0}\right\|_{\alpha}+L_{H} k_{0}+\|H(v)\|_{\alpha}\right)+C_{\alpha}\left(\frac{1-\gamma}{(1-\alpha) \beta-\gamma}\right)^{1-\gamma} T^{(1-\alpha) \beta-\gamma}\left\|\varphi_{k_{0}}\right\|_{L^{1 / \gamma}(0, T)} \\
+\frac{C_{\alpha} \tilde{k} T^{(1-\alpha) \beta}}{(1-\alpha) \beta}\left\|\phi_{k_{0}}\right\|_{L^{\infty}(0, T)} \leq k_{0} .
\end{gathered}
$$

Consider a mapping $\Gamma$ defined on $\Theta_{k_{0}}$ by

$$
\begin{aligned}
(\Gamma u)(t)= & \mathcal{S}_{\beta}(t)\left(u_{0}-H(u)\right)+\int_{0}^{t}(t-s)^{\beta-1} \mathcal{P}_{\beta}(t-s)\left(F\left(s, u(s), u\left(\kappa_{1}(s)\right)\right)\right. \\
& \left.\quad+\int_{0}^{s} K(s-\tau) G\left(\tau, u(\tau), u\left(\kappa_{2}(\tau)\right)\right) \mathrm{d} \tau\right) \mathrm{d} s \\
:= & \left(\Gamma_{1} u\right)(t)+\left(\Gamma_{2} u\right)(t), \quad t \in[0, T]
\end{aligned}
$$

It is easy to verify that $(\Gamma u)(.) \in C\left([0, T] ; X_{\alpha}\right)$ for every $u \in \Theta_{k_{0}}$. Moreover, for every pair $v, u \in \Theta_{k_{0}}$ and $t \in[0, T]$, by $\left(H_{1}\right)$ a direct calculation yields

$$
\begin{aligned}
\| & \left(\Gamma_{1} v\right)(t)+\left(\Gamma_{2} u\right)(t) \|_{\alpha} \\
\leq & \left\|\mathcal{S}_{\beta}(t)\left(u_{0}-H(v)\right)\right\|_{\alpha}+\int_{0}^{t}(t-s)^{\beta-1}\left\|A^{\alpha} \mathcal{P}_{\beta}(t-s)\right\|_{L(X)} \| F\left(s, u(s), u\left(\kappa_{1}(s)\right)\right) \\
& +\int_{0}^{s} K(s-\tau) G\left(\tau, u(\tau), u\left(\kappa_{2}(\tau)\right)\right) \mathrm{d} \tau \| \mathrm{d} s \\
\leq & M\left(\left\|u_{0}\right\|_{\alpha}+L_{H} k_{0}+\|H(v)\|_{\alpha}\right) \\
& +C_{\alpha} \int_{0}^{t}(t-s)^{\beta(1-\alpha)-1}\left(\varphi_{k_{0}}(s)+\tilde{k}\left\|\phi_{k_{0}}\right\|_{L^{\infty}(0, T)}\right) \mathrm{d} s \\
\leq & M\left(\left\|u_{0}\right\|_{\alpha}+L_{H} k_{0}+\|H(v)\|_{\alpha}\right) \\
& +C_{\alpha}\left(\frac{1-\gamma}{(1-\alpha) \beta-\gamma}\right)^{1-\gamma} T^{(1-\alpha) \beta-\gamma}\left\|\varphi_{k_{0}}\right\|_{L^{1 / \gamma}(0, T)} \frac{C_{\alpha} \tilde{k} T^{(1-\alpha) \beta}}{(1-\alpha) \beta}\left\|\phi_{k_{0}}\right\|_{L^{\infty}(0, T)} \\
\leq & k_{0} .
\end{aligned}
$$


That is, $\Gamma_{1} v+\Gamma_{2} u \in \Theta_{k_{0}}$ for every pair $v, u \in \Theta_{k_{0}}$. Therefore, the fractional Cauchy problem (1.1) has a mild solution if and only if the operator equation $\Gamma_{1} u+\Gamma_{2} u=u$ has a solution in $\Theta_{k_{0}}$.

In what follows, we will show that $\Gamma_{1}$ and $\Gamma_{2}$ satisfy the conditions of Lemma 2.5 . From $\left(H_{3}\right)$ and (3.1), we infer that $\Gamma_{1}$ is a contraction. Next, we show that $\Gamma_{2}$ is completely continuous on $\Omega_{k_{0}}$.

We first prove that $\Gamma_{2}$ is continuous on $\Theta_{k_{0}}$. Let $\left\{u_{n}\right\}_{n=1}^{\infty} \subset \Theta_{k_{0}}$ be a sequence such that $u_{n} \rightarrow u$ as $n \rightarrow \infty$ in $C\left([0, T] ; X_{\alpha}\right)$. Therefore, it follows from the continuity of $F$, $G, \kappa_{1}$ and $\kappa_{2}$ that for each $t \in[0, T]$,

$$
\begin{aligned}
& F\left(t, u_{n}(t), u_{n}\left(\kappa_{1}(t)\right)\right) \rightarrow F\left(t, u(t), u\left(\kappa_{1}(t)\right)\right) \quad \text { as } n \rightarrow \infty \\
& G\left(t, u_{n}(t), u_{n}\left(\kappa_{1}(t)\right)\right) \rightarrow G\left(t, u(t), u\left(\kappa_{2}(t)\right)\right) \quad \text { as } n \rightarrow \infty
\end{aligned}
$$

Also, by $\left(H_{1}\right)$, we see

$$
\begin{aligned}
& \int_{0}^{t}(t-s)^{\beta-1-\alpha \beta}\left\|F\left(s, u_{n}(s), u_{n}\left(\kappa_{1}(s)\right)\right)-F\left(s, u(s), u\left(\kappa_{1}(s)\right)\right)\right\| \mathrm{d} s \\
& \leq 2 \int_{0}^{t}(t-s)^{\beta-1-\alpha \beta} \varphi_{k_{0}}(s) \mathrm{d} s \\
& \leq 2\left(\frac{1-\gamma}{(1-\alpha) \beta-\gamma}\right)^{1-\gamma} T^{(1-\alpha) \beta-\gamma}\left\|\varphi_{k_{0}}\right\|_{L^{1 / \gamma}(0, T),}
\end{aligned}
$$

and

$$
\begin{aligned}
& \int_{0}^{t}(t-s)^{\beta-1-\alpha \beta} \int_{0}^{s} K(s-\tau) \| G\left(\tau, u_{n}(\tau), u_{n}\left(\kappa_{2}(\tau)\right)\right) \\
& \quad-G\left(\tau, u(\tau), u\left(\kappa_{2}(\tau)\right)\right) \| \mathrm{d} \tau \mathrm{d} s \\
& \leq 2 \widetilde{k}\left\|\phi_{k_{0}}\right\|_{L^{\infty}(0, T)} \int_{0}^{t}(t-s)^{\beta-1-\alpha \beta} \mathrm{d} s \\
& \leq \frac{2 \widetilde{k} T^{(1-\alpha) \beta}}{(1-\alpha) \beta}\left\|\phi_{k_{0}}\right\|_{L^{\infty}(0, T)}
\end{aligned}
$$

Hence, as

$$
\begin{aligned}
& \left\|\left(\Gamma_{2} u_{n}\right)(t)-\left(\Gamma_{2} u\right)(t)\right\|_{\alpha} \\
& \leq C_{\alpha} \int_{0}^{t}(t-s)^{\beta-1-\alpha \beta}\left\|F\left(s, u_{n}(s), u_{n}\left(\kappa_{1}(s)\right)\right)-F\left(s, u(s), u\left(\kappa_{1}(s)\right)\right)\right\| \mathrm{d} s \\
& +C_{\alpha} \int_{0}^{t}(t-s)^{\beta-1-\alpha \beta} \int_{0}^{s} K(s-\tau) \| G\left(\tau, u_{n}(\tau), u_{n}\left(\kappa_{2}(\tau)\right)\right) \\
& \quad-G\left(\tau, u(\tau), u\left(\kappa_{2}(\tau)\right)\right) \| \mathrm{d} \tau \mathrm{d} s,
\end{aligned}
$$

we conclude, using the Lebesgue dominated convergence theorem, that for all $t \in[0$, $T]$,

$$
\left\|\left(\Gamma_{2} u_{n}\right)(t)-\left(\Gamma_{2} u\right)(t)\right\|_{\alpha} \rightarrow 0, \quad \text { as } n \rightarrow \infty,
$$

which implies that

$$
\left|\Gamma_{2} u_{n}-\Gamma_{2} u\right|_{\alpha} \rightarrow 0, \quad \text { as } n \rightarrow \infty \text {. }
$$

This proves that $\Gamma_{2}$ is continuous on $\Theta_{k_{0}}$. It suffice to prove that $\Gamma_{2}$ is compact on $\Omega_{k_{0}}$. For the sake of brevity, we write

$$
\mathcal{N}(t, u(t))=F\left(t, u(t), u\left(\kappa_{1}(t)\right)\right)+\int_{0}^{t} K(t-\tau) G\left(\tau, u(\tau), u\left(\kappa_{2}(\tau)\right)\right) \mathrm{d} \tau
$$


Let $t \in[0, T]$ be fixed and $\varepsilon, \varepsilon_{1}>0$ be small enough. For $u \in \Omega_{k_{0}}$, we define the map $\Gamma^{\varepsilon, \varepsilon_{1}}$ by

$$
\begin{aligned}
\left(\Gamma^{\varepsilon, \varepsilon_{1}} u\right)(t) & =\int_{0}^{t-\varepsilon} \int_{\varepsilon_{1}}^{\infty} \beta \tau \Psi_{\beta}(\tau) T\left((t-s)^{\beta} \tau\right) \mathcal{N}(s, u(s)) \mathrm{d} \tau \mathrm{d} s \\
& =T\left(\varepsilon^{\beta} \varepsilon_{1}\right) \int_{0}^{t-\varepsilon} \int_{\varepsilon_{1}}^{\infty} \beta \tau \Psi_{\beta}(\tau) T\left((t-s)^{\beta} \tau-\varepsilon^{\beta} \varepsilon_{1}\right) \mathcal{N}(s, u(s)) \mathrm{d} \tau \mathrm{d} s .
\end{aligned}
$$

Therefore, from Lemma 2.1 we see that for each $t \in(0, T]$, the set $\left.\left\{\Gamma^{\varepsilon, \varepsilon_{1}} u\right)(t) ; u \in \Omega_{k_{0}}\right\}$ is relatively compact in $X_{\alpha}$. Then, as

$$
\begin{aligned}
& \left\|\left(\Gamma_{2} u\right)(t)-\left(\Gamma^{\varepsilon_{1} \varepsilon_{1}} u\right)(t)\right\|_{\alpha} \\
& \leq\left\|\int_{0}^{t} \int_{0}^{\varepsilon_{1}} \beta \tau(t-s)^{\beta-1} \Psi_{\beta}(\tau) T\left((t-s)^{\beta} \tau\right) \mathcal{N}(s, u(s)) \mathrm{d} \tau \mathrm{d} s\right\|_{\alpha} \\
& +\left\|\int_{t-\varepsilon}^{t} \int_{\varepsilon_{1}}^{\infty} \beta \tau(t-s)^{\beta-1} \Psi_{\beta}(\tau) T\left((t-s)^{\beta} \tau\right) \mathcal{N}(s, u(s)) \mathrm{d} \tau \mathrm{d} s\right\|_{\alpha} \\
& \leq \beta M_{\alpha}\left[\int_{0}^{t}(t-s)^{\beta(1-\alpha)-1}\left(\varphi_{k_{0}}(s)+\widetilde{k}\left\|\phi_{k_{0}}\right\|_{L^{\infty}(0, T)}\right) \mathrm{d} s \int_{0}^{\varepsilon_{1}} \tau^{1-\alpha} \Psi_{\beta}(\tau) \mathrm{d} \tau\right. \\
& \left.+\int_{t-\varepsilon}^{t}(t-s)^{\beta(1-\alpha)-1}\left(\varphi_{k_{0}}(s)+\widetilde{k}\left\|\phi_{k_{0}}\right\|_{L^{\infty}(0, T)}\right) \mathrm{d} s \int_{\varepsilon_{1}}^{\infty} \tau^{1-\alpha} \Psi_{\beta}(\tau) \mathrm{d} \tau\right] \\
& \leq \beta M_{\alpha}\left[\left(\frac{1-\gamma}{(1-\alpha) \beta-\gamma}\right)^{1-\gamma} T^{(1-\alpha) \beta-\gamma}\left\|\varphi_{k_{0}}\right\|_{L^{1 / \gamma}(0, T)}\right. \\
& \left.+\frac{\widetilde{k} T^{(1-\alpha) \beta}}{(1-\alpha) \beta}\left\|\phi_{k_{0}}\right\|_{L^{\infty}(0, T)}\right] \int_{0}^{\varepsilon_{1}} \tau^{1-\alpha} \Psi_{\beta}(\tau) \mathrm{d} \tau \\
& +\frac{\beta M_{\alpha} \Gamma(2-\alpha)}{\Gamma(1+\beta(1-\alpha))}\left[\left(\frac{1-\gamma}{(1-\alpha) \beta-\gamma}\right)^{1-\gamma}\left\|\varphi_{k_{0}}\right\|_{L^{1 / \gamma}(0, T)} \varepsilon^{(1-\alpha) \beta-\gamma}\right. \\
& \left.+\frac{\tilde{k}}{(1-\alpha) \beta}\left\|\phi_{k_{0}}\right\|_{L^{\infty}(0, T) \varepsilon^{(1-\alpha) \beta}}\right] \\
& \rightarrow 0 \quad \text { as } \varepsilon, \varepsilon_{1} \rightarrow 0^{+}
\end{aligned}
$$

in view of (2.1), we conclude, using the total boundedness, that for each $t \in[0, T]$, the set $\left.\left\{\Gamma_{2} u\right)(t) ; u \in \Omega_{k_{0}}\right\}$ is relatively compact in $X_{\alpha}$.

On the other hand, for $0<t_{1}<t_{2} \leq T$ and $\varepsilon^{\prime}>0$ small enough, we have

$$
\left\|\left(\Gamma_{2} u\right)\left(t_{1}\right)-\left(\Gamma_{2} u\right)\left(t_{2}\right)\right\|_{\alpha} \leq A_{1}+A_{2}+A_{3}+A_{4},
$$

where

$$
\begin{aligned}
& A_{1}=\int_{t_{1}}^{t_{2}}\left(t_{2}-s\right)^{\beta-1-\alpha \beta}\|\mathcal{N}(s, u(s))\| \mathrm{d} s \\
& A_{2}=\int_{0}^{t_{1}-\varepsilon^{\prime}}\left(t_{1}-s\right)^{\beta-1}\left\|A^{\alpha} \mathcal{P}_{\beta}\left(t_{2}-s\right)-A^{\alpha} \mathcal{P}_{\beta}\left(t_{1}-s\right)\right\|_{\mathcal{L}(x)}\|\mathcal{N}(s, u(s))\| \mathrm{d} s, \\
& A_{3}=\int_{t_{1}-\varepsilon^{\prime}}^{t_{1}}\left(t_{1}-s\right)^{\beta-1}\left(\left(t_{2}-s\right)^{-\alpha \beta}+\left(t_{1}-s\right)^{-\alpha \beta}\right)\|\mathcal{N}(s, u(s))\| \mathrm{d} s, \\
& A_{4}=\int_{0}^{t_{1}}\left|\left(t_{2}-s\right)^{\beta-1}-\left(t_{1}-s\right)^{\beta-1}\right| \cdot\left(t_{2}-s\right)^{-\alpha \beta}\|\mathcal{N}(s, u(s))\| \mathrm{d} s .
\end{aligned}
$$


Therefore, it follows from $\left(H_{1}\right)$, Lemma 2.2, and Lemma 2.3 that

$$
\begin{aligned}
& A_{1} \leq C_{\alpha} \int_{t_{1}}^{t_{2}}\left(t_{2}-s\right)^{\beta-1-\alpha \beta}\left(\varphi_{k_{0}}(s)+\widetilde{k}\left\|\phi_{k_{0}}\right\|_{L^{\infty}(0, T)}\right) \mathrm{d} s \\
& \leq C_{\alpha}\left(\frac{1-\gamma}{(1-\alpha) \beta-\gamma}\right)^{1-\gamma}\left\|\varphi_{k_{0}}\right\|_{L^{1 / \gamma}(0, T)}\left(t_{2}-t_{1}\right)^{(1-\alpha) \beta-\gamma} \\
& +\frac{C_{\alpha} \widetilde{k}\left\|\phi_{k_{0}}\right\|_{L^{\infty}(0, T)}}{(1-\alpha) \beta}\left(t_{2}-t_{1}\right)^{(1-\alpha) \beta}, \\
& A_{2} \leq \sup _{s \in\left[0, t_{1}-\varepsilon^{\prime}\right]}\left\|A^{\alpha} \mathcal{P}_{\alpha}\left(t_{2}-s\right)-A^{\alpha} \mathcal{P}_{\alpha}\left(t_{1}-s\right)\right\|_{\mathcal{L}(x)} \\
& \times \int_{0}^{t_{1}-\varepsilon^{\prime}}\left(t_{1}-s\right)^{\beta-1}\left(\varphi_{k_{0}}(s)+\widetilde{k}\left\|\phi_{k_{0}}\right\|_{L^{\infty}(0, T)}\right) \mathrm{d} s \\
& \leq\left[\left(\frac{1-\gamma}{\beta-\gamma}\right)^{1-\gamma}\left\|\varphi_{k_{0}}\right\|_{L^{1 / \gamma}(0, T)}\left(t_{1}^{\frac{\beta-\gamma}{1-\gamma}}-\varepsilon^{\frac{\beta-\gamma}{1-\gamma}}\right)^{1-\gamma}\right. \\
& \left.+\frac{\widetilde{k}\left\|\phi_{k_{0}}\right\|_{L^{\infty}(0, T)}}{\beta}\left(t_{1}^{\beta}-\varepsilon^{\prime \beta}\right)\right] \\
& \times \sup _{s \in\left[0, t_{1}-\varepsilon^{\prime}\right]}\left\|A^{\alpha} \mathcal{P}_{\alpha}\left(t_{2}-s\right)-A^{\alpha} \mathcal{P}_{\alpha}\left(t_{1}-s\right)\right\|_{\mathcal{L}(x)}, \\
& A_{3} \leq C_{\alpha} \int_{t_{1}-\varepsilon^{\prime}}^{\substack{s \in\left[0, t_{1}-\varepsilon^{\prime}\right] \\
t_{1}}}\left(t_{1}-s\right)^{\beta-1}\left(\left(t_{2}-s\right)^{-\alpha \beta}+\left(t_{1}-s\right)^{-\alpha \beta}\right) \\
& \times\left(\varphi_{k_{0}}(s)+\widetilde{k}\left\|\phi_{k_{0}}\right\|_{L^{\infty}(0, T)}\right) \mathrm{d} s \\
& \leq 2 C_{\alpha} \int_{t_{1}-\varepsilon^{\prime}}^{t_{1}}\left(t_{1}-s\right)^{\beta-1-\alpha \beta}\left(\varphi_{k_{0}}(s)+\widetilde{k}\left\|\phi_{k_{0}}\right\|_{L^{\infty}(0, T)}\right) \mathrm{d} s \\
& \leq C_{\alpha}\left(\frac{1-\gamma}{(\underset{\sim}{1}-\alpha) \beta-\gamma}\right)^{1-\gamma}\left\|\varphi_{k_{0}}\right\|_{L^{1 / \gamma}(0, T)} \varepsilon^{\varepsilon^{(1-\alpha) \beta-\gamma}} \\
& +\frac{C_{\alpha} \widetilde{k}\left\|\phi_{k_{0}}\right\|_{L^{\infty}(0, T)}}{(1-\alpha) \beta} \varepsilon^{(1-\alpha) \beta}, \\
& A_{4} \leq C_{\alpha} \int_{0}^{t_{1}}\left(\left(t_{1}-s\right)^{\beta-1}-\left(t_{2}-s\right)^{\beta-1}\right)\left(t_{2}-s\right)^{-\alpha \beta} \\
& \times\left(\varphi_{k_{0}}(s)+\tilde{k}\left\|\phi_{k_{0}}\right\|_{L^{\infty}(0, T)}\right) \mathrm{d} s \\
& \leq C_{\alpha} \int_{0}^{t_{1}}\left(\left(t_{1}-s\right)^{(1-\alpha) \beta-1}-\left(t_{2}-s\right)^{(1-\alpha) \beta-1}\right)\left(\varphi_{k_{0}}(s)+\widetilde{k}\left\|\phi_{k_{0}}\right\|_{L^{\infty}(0, T)}\right) \mathrm{d} s \\
& \leq C_{\alpha}\left(\frac{1-\gamma}{(1-\alpha) \beta-\gamma}\right)^{1-\gamma}\left\|\varphi_{k_{0}}\right\|_{L^{1 / \gamma}(0, T)} \\
& \times\left[t_{1}^{(1-\alpha) \beta-\gamma}-\left(t_{2}^{\frac{(1-\alpha) \beta-\gamma}{1-\gamma}}-\left(t_{2}-t_{1}\right)^{\frac{(1-\alpha) \beta-\gamma}{1-\gamma}}\right)^{1-\gamma}\right] \\
& +\frac{2 \widetilde{k}}{(1-\alpha) \beta}\left\|\phi_{k_{0}}\right\|_{L^{\infty}(0, T)}\left(t_{1}^{(1-\alpha) \beta}-t_{2}{ }^{(1-\alpha) \beta}+\left(t_{2}-t_{1}\right)^{(1-\alpha) \beta}\right) \text {, }
\end{aligned}
$$

from which it is easy to see that $A_{i}(i=1,2,3,4)$ tends to zero independently of $u \in \Omega_{k_{0}}$ as $t_{2}-t_{1} \rightarrow 0$ and $\varepsilon^{\prime} \rightarrow 0$. Hence, we can conclude that

$$
\left\|\left(\Gamma_{2} u\right)\left(t_{1}\right)-\left(\Gamma_{2} u\right)\left(t_{2}\right)\right\|_{\alpha} \rightarrow 0, \quad \text { as } t_{2}-t_{1} \rightarrow 0,
$$

and the limit is independently of $u \in \Omega_{k_{0}}$.

For the case when $0=t_{1}<t_{2} \leq T$, since

$$
\begin{aligned}
& \left\|\left(\Gamma_{2} u\right)\left(t_{1}\right)-\left(\Gamma_{2} u\right)\left(t_{2}\right)\right\|_{\alpha} \\
& =\left\|\int_{0}^{t_{2}}\left(t_{2}-s\right)^{\beta-1} \mathcal{P}_{\beta}\left(t_{2}-s\right) \mathcal{N}(s, u(s)) \mathrm{d} s\right\|_{\alpha} \\
& \leq C_{\alpha} \int_{0}^{t_{2}}\left(t_{2}-s\right)^{\beta-1-\alpha \beta}\left(\varphi_{k_{0}}(s)+\widetilde{k}\left\|\phi_{k_{0}}\right\|_{L^{\infty}(0, T)}\right) \mathrm{d} s \\
& \leq C_{\alpha}\left(\frac{1-\gamma}{(1-\alpha) \beta-\gamma}\right)^{1-\gamma}\left\|\varphi_{k}\right\|_{L^{1 / \gamma}(0, T)} t_{2}(1-\alpha) \beta-\gamma+\frac{C_{\alpha} \widetilde{k}\left\|\phi_{k_{0}}\right\|_{L^{\infty}(0, T)}}{(1-\alpha) \beta} t_{2}(1-\alpha) \beta .
\end{aligned}
$$

$\left\|\left(\Gamma_{2} u\right)\left(t_{1}\right)-\left(\Gamma_{2} u\right)\left(t_{2}\right)\right\|_{\alpha}$ can be made small when $t_{2}$ is small independently of $u \in \Omega_{k_{0}}$. Consequently, the set $\left\{\left(\Gamma_{2}\right)(\cdot) ; \cdot \in[0, T], u \in \Omega_{k_{0}}\right\}$ is equicontinuous. Now applying the Arzela-Ascoli theorem, it follows that $\Gamma_{2}$ is compact on $\Omega_{k_{0}}$. 
Therefore, applying Lemma 2.5 , we conclude that $\Gamma$ has a fixed point, which gives rise to a mild solution of Cauchy problem (1.1). This completes the proof.

The second result of this paper is the following theorem.

Theorem 3.2. Let the assumptions $\left(H_{0}\right),\left(H_{2}\right),\left(H_{4}\right)$ and $\left(H_{5}\right)$ be satisfied. Then, for $u_{0}$ $\in X_{\infty}$, the fractional Cauchy problem (1.1) has at least one mild solution provided that

$$
M \mu+\frac{2 C_{\alpha} T^{(1-\alpha) \beta}\left(L_{F}+\tilde{k} L_{G}\right)}{(1-\alpha) \beta}<1 .
$$

Proof. The proof is divided into the following two steps.

Step 1. Assume that $w \in C\left([\eta, T] ; X_{\alpha}\right)$ is fixed and set

$$
\widetilde{w}(t)= \begin{cases}w(t), \quad t \in[\eta, T] \\ w(\eta), \quad t \in[0, \eta]\end{cases}
$$

It is clear that $w \in C\left([0, T] ; X_{\alpha}\right)$. We define a mapping $\Gamma_{w}$ on $C\left([0, T] ; X_{\alpha}\right)$ by

$$
\begin{aligned}
\left(\Gamma_{w} u\right)(t)= & \mathcal{S}_{\beta}(t)\left(u_{0}-H(\widetilde{w})\right)+\int_{0}^{t}(t-s)^{\beta-1} \mathcal{P}_{\beta}(t-s)\left(F\left(s, u(s), u\left(\kappa_{1}(s)\right)\right)\right. \\
& \left.+\int_{0}^{s} K(s-\tau) G\left(\tau, u(\tau), u\left(\kappa_{2}(\tau)\right)\right) \mathrm{d} \tau\right) \mathrm{d} s, \quad t \in[0, T] .
\end{aligned}
$$

Clearly, $\left(\Gamma_{w} u\right)(\cdot) \in C\left([0, T] ; X_{\alpha}\right)$ for every $u \in C\left([0, T] ; X_{\alpha}\right)$. Moreover, for $u \in \Theta_{k}$, from $\left(H_{2}\right)$, it follows that

$$
\begin{aligned}
& \left\|\left(\Gamma_{w} u\right)(t)\right\|_{\alpha} \\
& \leq\left\|\mathcal{S}_{\beta}(t)\left(u_{0}-H(\widetilde{w})\right)\right\|_{\alpha} \\
& \quad+\int_{0}^{t}(t-s)^{\beta-1} \| \mathcal{P}_{\beta}(t-s)\left(F\left(s, u(s), u\left(\kappa_{1}(s)\right)\right)\right. \\
& \left.\quad+\int_{0}^{s} K(s-\tau) G\left(\tau, u(\tau), u\left(\kappa_{2}(\tau)\right)\right) \mathrm{d} \tau\right) \|_{\alpha} \mathrm{d} s \\
& \leq M\left(\left\|u_{0}\right\|_{\alpha}+\|H(\widetilde{w})\|_{\alpha}\right) \\
& \quad+C_{\alpha} \int_{0}^{t}(t-s)^{\beta(1-\alpha)-1}\left[L_{F}\left(\|u(s)\|_{\alpha}+\left\|u\left(\kappa_{1}(s)\right)\right\|_{\alpha}\right)+\|F(s, v, v)\|\right. \\
& \left.\quad+L_{G} \int_{0}^{s} K(s-\tau)\left(\|u(\tau)\|_{\alpha}+\left\|u\left(\kappa_{2}(\tau)\right)\right\|_{\alpha}+\|G(s, v, v)\|\right) \mathrm{d} \tau\right] \mathrm{d} s \\
& \leq M\left(\left\|u_{0}\right\|_{\alpha}+\|H(\widetilde{w})\|_{\alpha}\right)+\frac{2 k C_{\alpha}\left(L_{F}+\widetilde{k} L_{G}\right) t^{(1-\alpha) \beta}}{(1-\alpha) \beta} \\
& \quad+\frac{C_{\alpha}\left(\max _{0 \leq s \leq T}\|F(s, v, v)\|+\widetilde{k} \max _{0 \leq s \leq T}\|G(s, v, v)\|\right) T^{(1-\alpha) \beta}}{(1-\alpha) \beta},
\end{aligned}
$$

where $v \in C\left([0, T] ; X_{\alpha}\right)$ is fixed with $|v|_{\alpha} \equiv 0$, which implies that there exists a integer $k_{0}>0$ such that $\Gamma_{w}$ maps $\Theta_{k_{0}}$ into itself. In fact, if this is not the case, then for each $k>0$, there would exist $u_{k} \in \Theta_{k}$ and $t_{k} \in[0, T]$ such that $\left\|\left(\Gamma_{w} u_{k}\right)\left(t_{k}\right)\right\|_{\alpha}>k$. Thus, we have

$$
\begin{aligned}
k<\| & \left\|\left(\Gamma_{w} u_{k}\right)\left(t_{k}\right)\right\|_{\alpha} \leq M\left(\left\|u_{0}\right\|_{\alpha}+\|H(\widetilde{u})\|_{\alpha}\right)+\frac{2 k C_{\alpha}\left(L_{F}+\widetilde{k} L_{G}\right) T^{(1-\alpha) \beta}}{(1-\alpha) \beta} \\
& +\frac{C_{\alpha}\left(\max _{0 \leq s \leq T}\|F(s, v, v)\|+\widetilde{k} \max _{0 \leq s \leq T}\|G(s, v, v)\|\right) T^{(1-\alpha) \beta}}{(1-\alpha) \beta}
\end{aligned}
$$


Dividing on both sides by $k$ and taking the lower limit as $k \rightarrow+\infty$, we get

$$
1 \leq \frac{2 C_{\alpha}\left(L_{F}+\tilde{k} L_{G}\right) T^{(1-\alpha) \beta}}{(1-\alpha) \beta}
$$

this contradicts (3.2). Also, for $u, v \in \Theta_{k_{0}}$, a direct calculation yields

$$
\begin{aligned}
& \left\|\left(\Gamma_{w} u\right)(t)-\left(\Gamma_{w} v\right)(t)\right\|_{\alpha} \\
& =\| \int_{0}^{t}(t-s)^{\beta-1} \mathcal{P}_{\beta}(t-s)\left[F\left(s, u(s), u\left(\kappa_{1}(s)\right)\right)-F\left(s, v(s), v\left(\kappa_{1}(s)\right)\right)\right. \\
& \left.\quad+\int_{0}^{s} K(s-\tau)\left(G\left(\tau, u(\tau), u\left(\kappa_{2}(\tau)\right)\right)-G\left(\tau, v(\tau), v\left(\kappa_{2}(\tau)\right)\right)\right) \mathrm{d} \tau\right] \mathrm{d} s \|_{\alpha} \\
& \leq C_{\alpha} \int_{0}^{t}(t-s)^{\beta-1-\alpha \beta}\left[\left\|F\left(s, u(s), u\left(\kappa_{1}(s)\right)\right)-F\left(s, v(s), v\left(\kappa_{1}(s)\right)\right)\right\| \mathrm{d} s\right. \\
& \left.\quad+\int_{0}^{s} K(s-\tau)\left\|G\left(\tau, u(\tau), u\left(\kappa_{2}(\tau)\right)\right)-G\left(\tau, v(\tau), v\left(\kappa_{2}(\tau)\right)\right)\right\| \mathrm{d} \tau\right] \mathrm{d} s \\
& \leq C_{\alpha} \int_{0}^{t}(t-s)^{\beta-1-\alpha \beta}\left[L_{F}\left(\|u(s)-v(s)\|_{\alpha}+\left\|u\left(\kappa_{1}(s)\right)-v\left(\kappa_{1}(s)\right)\right\|_{\alpha}\right)\right. \\
& \left.\quad+L_{G} \int_{0}^{s} K(s-\tau)\left(\|u(\tau)-v(\tau)\|_{\alpha}+\left\|u\left(\kappa_{2}(\tau)\right)-v\left(\kappa_{2}(\tau)\right)\right\|_{\alpha}\right) \mathrm{d} \tau\right] \mathrm{d} s \\
& \leq \frac{2 C_{\alpha} T^{(1-\alpha) \beta}\left(L_{F}+\widetilde{k} L_{G}\right)}{(1-\alpha) \beta}|u-v|_{\alpha},
\end{aligned}
$$

which together with (3.2) implies that $\Gamma_{w}$ is a contraction mapping on $\Theta_{k_{0}}$. Thus, by the Banach contraction mapping principle, $\Gamma_{w}$ has a unique fixed point $u_{w} \in \Theta_{k_{0}}$, i.e.,

$$
\begin{aligned}
u_{w}= & \mathcal{S}_{\beta}(t)\left(u_{0}-H(\widetilde{w})\right)+\int_{0}^{t}(t-s)^{\beta-1} \mathcal{P}_{\beta}(t-s)\left(F\left(s, u_{w}(s), u_{w}\left(\kappa_{1}(s)\right)\right)\right. \\
& \left.+\int_{0}^{s} K(s-\tau) G\left(\tau, u_{w}(\tau), u_{w}\left(\kappa_{2}(\tau)\right)\right) \mathrm{d} \tau\right) \mathrm{d} s
\end{aligned}
$$

for $t \in[0, T]$.

Step 2. Write

$$
\Theta_{k_{0}}^{\eta}=\left\{u \in C\left([\eta, T] ; X_{\alpha}\right) ;\|u(t)\|_{\alpha} \leq k_{0} \quad \text { for all } t \in[\eta, T]\right\} .
$$

It is clear that $\Theta_{k_{0}}^{\eta}$ is a bounded closed convex subset of $C\left([\eta, T] ; X_{\alpha}\right)$.

Based on the argument in Step 1, we consider a mapping $\mathcal{F}$ on $\Theta_{k_{0}}^{\eta}$ defined by

$$
(\mathcal{F} w)(t)=u_{w}, \quad t \in[\eta, T] .
$$

It follows from $\left(H_{5}\right)$ and (3.2) that $\mathcal{F}$ maps $\Theta_{k_{0}}^{\eta}$ into itself. Moreover, for $w_{1}, w_{2} \in \Theta_{k_{0}}^{\eta}$ from Step 1, we have

$$
\left(1-\frac{2 C_{\alpha} T^{(1-\alpha) \beta}\left(L_{F}+\tilde{k} L_{G}\right)}{(1-\alpha) \beta}\right)\left|u_{w_{1}}-u_{w_{2}}\right|_{\alpha} \leq M\left\|H\left(\bar{w}_{1}\right)-H\left(\bar{w}_{2}\right)\right\|_{\alpha \prime}
$$

that is,

$$
\sup _{t \in[\eta, T]}\left\|\mathcal{F} w_{1}(t)-\mathcal{F} w_{2}(t)\right\|_{\alpha} \rightarrow 0 \quad \text { as } w_{1} \rightarrow w_{2} \text { in } \Theta_{k_{0}}^{\eta}
$$


which yields that $\mathcal{F}$ is continuous. Next, we prove that $\mathcal{F}$ has a fixed point in $\Theta_{k_{0}}^{\eta}$. It will suffice to prove that $\mathcal{F}$ is a compact operator. Then, the result follows from Lemma 2.6.

Let's decompose the mapping $\mathcal{F}=\mathcal{F}_{1}+\mathcal{F}_{2}$ as

$$
\begin{aligned}
\left(\mathcal{F}_{1} w\right)(t)= & \mathcal{S}_{\beta}(t)\left(u_{0}-H(\tilde{w})\right), \\
\left(\mathcal{F}_{2} w\right)(t)= & \int_{0}^{t}(t-s)^{\beta-1} \mathcal{P}_{\beta}(t-s)\left(F\left(s, u_{w}(s), u_{w}\left(\kappa_{1}(s)\right)\right)\right. \\
& \left.+\int_{0}^{s} K(s-\tau) G\left(\tau, u_{w}(\tau), u_{w}\left(\kappa_{2}(\tau)\right)\right) \mathrm{d} \tau\right) \mathrm{d} s .
\end{aligned}
$$

Since assumption $\left(H_{5}\right)$ implies that the $\operatorname{set}\left\{H(\widetilde{w}) ; w \in \Theta_{k_{0}}^{\eta}\right\}$ is bounded in $X_{\alpha}$, it follows from Lemma 2.4 that for each $t \in[\eta, T],\left\{\left(\mathcal{F}_{1} w\right)(t) ; w \in \Theta_{k_{0}}^{\eta}\right\}$ is relatively compact in $X_{\alpha}$. Also, for $\eta \leq t_{1} \leq t_{2} \leq T$,

$$
\left\|\left(\mathcal{S}_{\beta}\left(t_{2}\right)-\mathcal{S}_{\beta}\left(t_{1}\right)\right)\left(u_{0}-H(\widetilde{w})\right)\right\|_{\alpha} \rightarrow 0 \quad \text { as } t_{2}-t_{1} \rightarrow 0
$$

independently of $w \in \Theta_{k_{0}}^{\eta}$. This proves that the set $\left\{\left(\mathcal{F}_{1} w\right)(\cdot) ; w \in \Theta_{k_{0}}^{\eta}\right\}$ is equicontinuous. Thus, an application of Arzela-Ascoli's theorem yields that $\mathcal{F}_{1}$ is compact.

Observe that the set

$$
\left\{F\left(t, u(t), u\left(\kappa_{1}(t)\right)\right)+\int_{0}^{t} K(t-\tau) G\left(\tau, u(\tau), u\left(\kappa_{2}(\tau)\right)\right) \mathrm{d} \tau ; t \in[0, T], w \in \Theta_{k_{0}}^{\eta}\right\}
$$

is bounded in $X$. Therefore, using Lemma 2.1, Lemma 2.2 and Lemma 2.3, it is not difficult to prove, similar to the argument with $\Gamma_{2}$ in Theorem 3.1, that $\mathcal{F}_{2}$ is compact. Hence, making use of Lemma 2.6 we conclude that $\mathcal{F}$ has a fixed point $w^{*} \in \Theta_{k_{0}}^{\eta}$. Put $q=u_{w^{*}}$. Then,

$$
\begin{aligned}
q(t)= & \mathcal{S}_{\beta}(t)\left(u_{0}-H\left(\widetilde{w^{*}}\right)\right)+\int_{0}^{t}(t-s)^{\beta-1} \mathcal{P}_{\beta}(t-s)\left(F\left(s, q(s), q\left(\kappa_{1}(s)\right)\right)\right. \\
& \left.\left.+\int_{0}^{s} K(s-\tau) G\left(\tau, q(\tau), q\left(\kappa_{2}(\tau)\right)\right) \mathrm{d} \tau\right)\right) \mathrm{d} s, \quad t \in[0, T] .
\end{aligned}
$$

Since $u_{w^{*}}=\mathcal{F} w^{*}=w^{*}(t \in[\eta, T]), H\left(w^{*}\right)=H(q)$ and hence $q$ is a mild solution of the fractional Cauchy problem (1.1). This completes the proof.

\section{Example}

In this section, we present an example to our abstract results, which do not aim at generality but indicate how our theorem can be applied to concrete problem.

We consider the partial differential equation with Dirichlet boundary condition and nonlocal initial condition in the form 


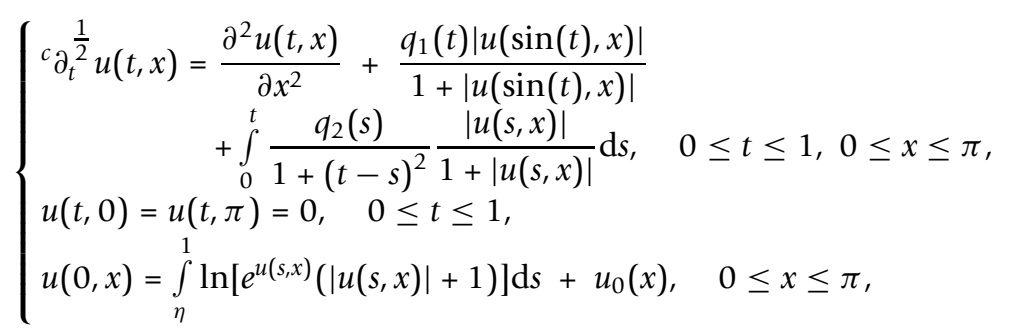

where the functions $q_{1}, q_{2}$ are continuous on $[0,1]$ and $0<\eta<1$.

Let $X=L^{2}[0, \pi]$ and the operators $A=\frac{\partial^{2}}{\partial x^{2}}: D(A) \subset X \mapsto X$ be defined by

$$
\begin{gathered}
D(A)=\left\{u \in X ; u, u^{\prime} \text { are absolutely continuous, } u^{\prime \prime} \in X\right. \text {, and } \\
u(0)=u(\pi)=0\} .
\end{gathered}
$$

Then, $A$ has a discrete spectrum and the eigenvalues are $-n^{2}, \mathrm{n} \in \mathbb{N}$, with the corresponding normalized eigenvectors $y_{n}(x)=\sqrt{\frac{2}{\pi}} \sin (n x)$. Moreover, $A$ generates a compact, analytic semigroup $\{T(t)\}_{t \geq 0}$. The following results are well also known (see [29] for more details):

(1) $T(t) u=\sum_{n=1}^{\infty} e^{-n^{2} t}\left(u, y_{n}\right) y_{n},\|T(t)\|_{\mathcal{L}(X)} \leq e^{-t}$ for all $t \geq 0$.

(2) $A^{-\frac{1}{2}} u=\sum_{n=1}^{\infty} \frac{1}{n}\left(u, y_{n}\right) y_{n}$ for each $u \in X$. In particular, $\left\|A^{-\frac{1}{2}}\right\|_{\mathcal{L}(X)}=1$.

(3) $A^{\frac{1}{2}} u=\sum_{n=1}^{\infty} n\left(u, y_{n}\right) y_{n}$ with the domain

$$
D\left(A^{\frac{1}{2}}\right)=\left\{u \in X ; \sum_{n=1}^{\infty} n\left(u, y_{n}\right) y_{n} \in X\right\} .
$$

Denote by $E_{\zeta, \beta}$, the generalized Mittag-Leffler special function (cf., e.g., [4]) defined by

$$
E_{\zeta, \beta}(t)=\sum_{k=0}^{\infty} \frac{t^{k}}{\Gamma(\zeta k+\beta)} \quad \zeta, \beta>0, \quad t \in \mathbb{R}
$$

Therefore, we have

$$
\begin{array}{lll}
\mathcal{S}_{\beta}(t) u=\sum_{n=1}^{\infty} E_{\beta}\left(-n^{2} t^{\beta}\right)\left(u, y_{n}\right) y_{n}, & u \in X ; & \left\|\mathcal{S}_{\beta}(t)\right\|_{\mathcal{L}(X)} \leq 1, \\
\mathcal{P}_{\beta}(t) u=\sum_{n=1}^{\infty} e_{\beta}\left(-n^{2} t^{\beta}\right)\left(u, y_{n}\right) y_{n}, & u \in X ; & \left\|\mathcal{P}_{\beta}(t)\right\|_{\mathcal{L}(X)} \leq \frac{\beta}{\Gamma(1+\beta)}
\end{array}
$$

for all $t \geq 0$, where $E_{\beta}(t):=E_{\beta, 1}(t)$ and $e_{\beta}(t):=E_{\beta, \beta}(t)$.

The consideration of this section also needs the following result.

Lemma 4.1. [31] If $w \in D\left(A^{\frac{1}{2}}\right.$, then $w$ is absolutely continuous, $w^{\prime} \in X$, and $\left\|w^{\prime}\right\|=\left\|A^{\frac{1}{2}} w\right\|$ 
Define

$$
\begin{aligned}
& F\left(t, u(t), u\left(\kappa_{1}(t)\right)\right)(x)=\frac{q_{1}(t)|u(\sin (t), x)|}{1+|u(\sin (t), x)|}, \\
& K(t)=\frac{1}{t^{2}+1}, \quad \kappa_{1}(t)=\sin (t), \quad \kappa_{2}(t)=t, \\
& G\left(t, u(t), u\left(\kappa_{2}(t)\right)\right)(x)=\frac{q_{2}(t)|u(t, x)|}{1+|u(t, x)|}, \\
& H(u)(x)=\int_{\eta}^{1} \ln \left[e^{u(s, x)}(|u(s, x)|+1)\right] \mathrm{d} s .
\end{aligned}
$$

Therefore, it is not difficult to verify that $F, G:[0,1] \times X_{\frac{1}{2}} \times X_{\frac{1}{2}} \rightarrow X$ and $H: C\left([0,1] ; X_{\frac{1}{2}}\right) \rightarrow X_{\frac{1}{2}}$ are continuous,

$$
\begin{aligned}
& \left\|F\left(t, u(t), u\left(\kappa_{1}(t)\right)\right)-F\left(t, v(t), v\left(\kappa_{1}(t)\right)\right)\right\| \\
& \leq \mu_{1}\left\|A^{-\frac{1}{2}}\right\|_{\mathcal{L}(X)}\left\|u\left(\kappa_{1}(t)\right)-v\left(\kappa_{1}(t)\right)\right\|_{\frac{1}{2}{ }^{\prime}} \\
& \left\|G\left(t, u(t), u\left(\kappa_{1}(t)\right)\right)-G\left(t, v(t), v\left(\kappa_{1}(t)\right)\right)\right\| \\
& \leq \mu_{2}\left\|A^{-\frac{1}{2}}\right\|_{\mathcal{L}(X)}\|u(t)-v(t)\|_{\frac{1}{2}{ }^{\prime}}
\end{aligned}
$$

where $\mu_{i}:=\sup _{t \in[0,1]}\left|q_{i}(t)\right|$, and for any $u$ satisfying $|u|_{\frac{1}{2}} \leq k$,

$$
\|H(u)\|_{\frac{1}{2}}=\left\|A^{\frac{1}{2}} H(u)(\cdot)\right\|=\left\|H(u)^{\prime}(\cdot)\right\| \leq 2(1-\eta) k
$$

in view of Lemma 4.1.

Now, we note that the problem (4.1) can be reformulated as the abstract problem (1.1) and the assumptions $\left(H_{0}\right),\left(H_{2}\right),\left(H_{4}\right)$ and $\left(H_{5}\right)$ hold with

$$
\alpha=\frac{1}{2}, \quad T=1, \quad L_{F}=\mu_{1}, \quad L_{G}=\mu_{2}, \quad \Phi(k)=2(1-\eta) k, \quad \mu=2(1-\eta) .
$$

Thus, when $1-\eta+4 M_{\frac{1}{2}}\left(\mu_{1}+\mu_{2}\right)<\frac{1}{2}$ such that condition (3.2) is verified, (4.1) has at least one mild solution due to Theorem 3.2.

\section{Acknowledgements}

We would like to thank the referees for their valuable comments and suggestions. This research was supported in part by NNSF of China (11101202), NSF of JiangXi Province of China (2009GQS0018), and Youth Foundation of JiangXi Provincial Education Department of China (GJJ10051).

\section{Authors' contributions}

All authors contributed equally to the manuscript and read and approved the final manuscript.

\section{Competing interests}

The authors declare that they have no competing interests.

Received: 17 December 2010 Accepted: 16 August 2011 Published: 16 August 2011

\section{References}

1. Anh Vo, V, Leonenko, NN: Spectral analysis of fractional kinetic equations with randomdata. J Stat Phys. 104, 1349-1387 (2001). doi:10.1023/A:1010474332598

2. Eidelman, SD, Kochubei, AN: Cauchy problem for fractional diffusion equations. J Differ Equ. 199(2), 211-255 (2004). doi:10.1016/j.je.2003.12.002

3. Metzler, R, Klafter, J: The random walk's guide to anomalous diffusion: a fractional dynamics approach. Phys Rep. 339 1-77 (2000). doi:10.1016/S0370-1573(00)00070-3

4. Miller, KS, Ross, B: An Introduction to the Fractional Calculus and Differential Equations. Wiley, New York (1993) 
5. Podlubny, I, Petraš, I, Vinagre, BM, O'Leary, P, Dorčak, L: Analogue realizations of fractional-order controllers: fractional order calculus and its applications. Nonlinear Dyn. 29, 281-296 (2002). doi:10.1023/A:1016556604320

6. Samko, SG, Kilbas, AA, Marichev, Ol: Fractional Integrals and Derivatives, Theory and Applications. Gordon and Breach, Yverdon (1993)

7. Mainardi, F: Fractional calculus: some basic problems in continuum and statistical mechanics. In: Carpinteri A, Mainardi F (eds.) Fractals and Fractional Calculus in Continuum Mechanics. pp. 291-348. Springer, New York (1997)

8. Agarwal, RP, Lakshmikantham, V, Nieto, JJ: On the concept of solution for fractional differential equations with uncertainty. Nonlinear Anal. 72, 2859-2862 (2010). doi:10.1016/j.na.2009.11.029

9. Agarwal, RP, Zhou, Y, He, YY: Existence of fractional neutral functional differential equations. Comput Math Appl. 59, 1095-1100 (2010)

10. Cuevas, C, de Souza, JC: S-asymptotically w-periodic solutions of semilinear fractional integro-differential equations. Appl Math Lett. 22, 865-870 (2009). doi:10.1016/j.aml.2008.07.013

11. Hernández, E, O'Regan, D, Balachandran, $\mathrm{K}:$ On recent developments in the theory of abstract differential equations with fractional derivatives. Nonlinear Anal. 73, 3462-3471 (2010). doi:10.1016/j.na.2010.07.035

12. Hilfer, H: Applications of Fractional Calculus in Physics. World Scientific Publ. Co., Singapure (2000)

13. Kilbas, AA, Srivastava, HM, Juan Trujillo, J: Theory and applications of fractional differential equations. In North-Holland Mathematics Studies, vol. 204,Elsevier, Amsterdam (2006)

14. Zhou, Y, Jiao, F: Nonlocal Cauchy problem for fractional evolution equations. Nonlinear Anal Real World Appl. 11, 4465-4475 (2010). doi:10.1016/j.nonrwa.2010.05.029

15. Zhou, Y, Jiao, F: Existence of mild solutions for fractional neutral evolution equations. Comput Math Appl. 59, 1063-1077 (2010)

16. Diagana, T, Mophou, GM, N'Guérékata, GM: On the existence of mild solutions to some semilinear fractional integrodifferential equations. E J Qual Theory Diff Equ. 58, 1-17 (2010)

17. Byszewski, L: Theorems about the existence and uniqueness of solutions of a semilinear evolution nonlocal Cauchy problem. J Math Anal Appl. 162, 494-505 (1991). doi:10.1016/0022-247X(91)90164-U

18. Byszewski, L, Lakshmikantham, V: Theorem about the existence and uniqueness of a solution of a nonlocal abstract Cauchy problem in a Banach space. Appl Anal. 40, 11-19 (1991). doi:10.1080/00036819008839989

19. Deng, K: Exponential decay of solutions of semilinear parabolic equations with nonlocal initial conditions. J Math Anal Appl. 179, 630-637 (1993). doi:10.1006/jmaa.1993.1373

20. Aizicovici, S, Staicu, V: Multivalued evolution equations with nonlocal initial conditions in Banach spaces. NoDEA Nonlinear Differ Equ Appl. 14, 361-376 (2007). doi:10.1007/s00030-007-5049-5

21. Ezzinbi, K, Fu, X, Hilal, K: Existence and regularity in the -norm for some neutral partial differential equations with nonlocal conditions. Nonlinear Anal. 67, 1613-1622 (2007). doi:10.1016/j.na.2006.08.003

22. Fan, Z: Impulsive problems for semilinear differential equations with nonlocal conditions. Nonlinear Anal. 72, 1104-1109 (2010). doi:10.1016/j.na.2009.07.049

23. Liang, J, van Casteren, J, Xiao, TJ: Nonlocal Cauchy problems for semilinear evolution equations. Nonlinear Anal. 50 173-189 (2002). doi:10.1016/50362-546X(01)00743-X

24. Liang, J, Liu, JH, Xiao, TJ: Nonlocal Cauchy problems governed by compact operator families. Nonlinear Anal. 57, 183-189 (2004). doi:10.1016/j.na.2004.02.007

25. Liang, J, Liu, JH, Xiao, TJ: Nonlocal impulsive problems for nonlinear differential equations in Banach spaces. Math Comput Model. 49, 798-804 (2009). doi:10.1016/j.mcm.2008.05.046

26. Liang, J, Xiao, TJ: Semilinear integrodifferential equations with nonlocal initial conditions. Comput Math Appl. 47, 863-875 (2004). doi:10.1016/50898-1221(04)90071-5

27. Liu, H, Chang, JC: Existence for a class of partial differential equations with nonlocal conditions. Nonlinear Anal. 70, 3076-3083 (2009). doi:10.1016/.na.2008.04.009

28. N'Guérékata, GM: A Cauchy problem for some fractional abstract differential equation with nonlocal conditions. Nonlinear Anal. 70, 1873-1876 (2009). doi:10.1016/j.na.2008.02.087

29. Pazy, A: Semigroups of Linear Operators and Applications to Partial Differential Equations. Springer, New York (1983)

30. Hernández, E, Rabelloa, M, Henriquez, HR: Existence of solutions for impulsive partial neutral functional differential equations. J Math Anal Appl. 331, 1135-1158 (2007). doi:10.1016/j.jmaa.2006.09.043

31. Travis, CC, Webb, GF: Partial functional differential equations with deviating arguments in the time variable. J Math Anal Appl. 56, 397-409 (1976). doi:10.1016/0022-247X(76)90052-4

doi:10.1186/1687-1847-2011-25

Cite this article as: Wang et al:: Abstract fractional integro-differential equations involving nonlocal initial

conditions in $\alpha$-norm. Advances in Difference Equations 2011 2011:25. 